\title{
Clustering and Communications Scheduling in WSN using Mixed Integer Linear Programming
}

\author{
François Avril, Thibault Bernard, Alain Bui and Devan Sohier
}

\begin{abstract}
We consider the problem of scheduling communications in Wireless Sensor Networks to ensure battery preservation through the use of sensors' sleeping mode. We propose a communication protocol in 1-hop WSNs and extend it to multi-hop WSNs thanks to a 1-hop clustering algorithm. Once the clustering is obtained, we propose to schedule communications in a virtual communication ring so as to avoid collisions. Since clusters are complete, only one sensor can speak or listen in a cluster at a time, and all sensors need to speak in each of their clusters at least once to achieve the communication protocol. We model this situation as a mathematical program.
\end{abstract}

\section{Introduction}

Sensors are tiny battery-powered devices that scan environmental parameters, and wirelessly communicate between them to monitor phenomena pertaining to a wide range: environmental issues, fire disasters, military applications,... Monitored data can then be relayed to a base station (BS) in charge of analysing it and triggering an alarm if needed.

Wireless Sensor Networks (WSN) raise several issues: Routing (i.e. how to transmit efficiently the scanned data to the base station), Security (i.e. how to ensure that scanned data remain anonymous and can not be used by a third party) and Powersaving (i.e. how to reduce battery consumption and increase the lifetime of each device).

In this paper, we focus on the third issue (while ensuring communications in the WSN): saving power and increasing the network lifetime. To fulfill this goal, several papers have focused on techniques to relay data efficiently in order to reduce energy consumption [20], [3] or to guarantee that messages are delivered to their recipients [2], [13]. We focus on collision avoidance: as shown in [8], most energy is consumed by communications between sensors. A collision causes the sensor to transmit the message again, which wastes energy. In order to minimize collisions, the Wake-up/Sleep mechanisms and/or the control messages RTS/CTS/ACK defined in 802.11x standards, are used to design energy-efficient MAC protocols for WSN as those described in T-MAC [17], Z-MAC [14], X-MAC and B-

François Avril is with the CaRO Team PRiSM CNRS, Université de Versailles Saint-Quentin-en-Yvelines, 45, av. des Etats Unis F-78035 Versailles Cedex, France, email: francois.avril@prism.uvsq.fr.

Thibault Bernard is with the CaRO Team PRiSM CNRS, Université de Versailles Saint-Quentin-en-Yvelines, 45, av. des Etats Unis F-78035 Versailles Cedex , France and with SYSCOM Team CReSTIC, Université de Reims Champagne Ardenne F-51687 Reims, cedex, France, email: thibault.bernard@univreims.fr.

Alain Bui is with the CaRO Team PRiSM CNRS, Université de Versailles Saint-Quentin-en-Yvelines, 45, av. des Etats Unis F-78035 Versailles Cedex, France, email: alain.bui@ prism.uvsq.fr.

Devan Sohier is with the CaRO Team PRiSM CNRS, Universite de Versailles Saint-Quentin-en-Yvelines, 45, av. des Etats Unis F-78035 Versailles Cedex, France, email: devan.sohier@prism.uvsq.fr.

\section{MAC[7]}

In this paper, we propose to save battery power by scheduling communications so that sensors spend as much time as possible in their sleep state. In [5], [6], we have proposed to structure communications among all sensors in a 1-hop network in such a way that no collision is possible. We propose to make sensors of a cluster communicate in a (virtual) token ring so that only one sensor sends data at a time, and only one other sensor receives them (i.e. all other sensors are in the sleep state). In [1], we have proposed a preliminary clustering procedure to extend this protocol to multihop WSN.

In this paper, we propose a complete clustering procedure that divides the WSN in 1-hop clusters and investigate on scheduling the discussion among all clusters to avoid collisions, in particular on gateway sensors (sensors belonging to several clusters).

The paper is organized as follows, Section II presents related works about energy preservation in WSN. In Section III, we present the model used to build the scheduling solution and previous works on which our solution rely. In Section IV, we define an adequate clustering and give an algorithm to split a multihop WSN into 1-hop clusters. A complete example of a correct scheduling is given Section V. In Section VI, a mixed integer linear programming description of the scheduling problem is presented and some optimization issues are analyzed. Concluding remarks and future works are given in Section VII.

\section{Related Works}

Battery preservation in WSN is a major issue, and many solutions have been proposed. In [8], the authors detail the energy consumption (cf. Fig. 1) of sensors depending on their state. They have shown that power is mainly spent by communications between sensors.

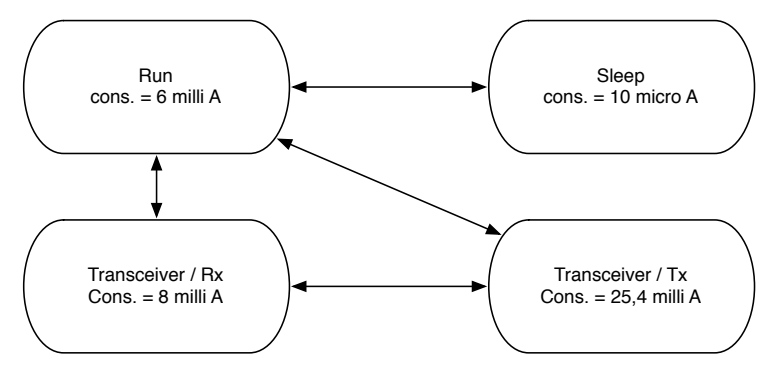

Fig. 1. Energy consumption of Mica 2 sensor through different states

Thus maximizing the time spent in the sleep mode preserves battery and increases sensor lifetime. This also avoids lots of collisions that are expensive in terms of battery consumption. The protocol we propose, aims at increasing the time spent in sleeping mode and avoiding collisions. 
In [9], the authors propose to maximize lifetime of a nonmobile WSN through routing procedures. They formulate the routing problem as a linear programming problem, where the objective is to maximize the network lifetime. Their algorithm is based on the link cost of communication between sensors: they build shortest paths for routing purpose. They save energy consumption by considering the information flow between sensors. In our solution, we do not focus on the size of the transmitted information but on the discussion scheduling over the sensors.

In [4], the authors minimize power use by reducing the transmission power of each sensor. As the consumed power increases quadratically with the transmission range, they build an approximation of a connected minimal weighted dominated set, use it as a backbone over sensors (by reducing transmission radius), and ensure the connectivity of the whole network. Such a solution could be applied as a preliminary phase to our solution.

The problem of synchronisation in WSNs has been investigated in [16]. The authors precise: "Existing time synchronisation methods were not designed with wireless sensor networks in mind, and need to be extended or redesigned." WSNs use synchronisation very often: TDMA schedule, data fusion, synchronized sleep period. They design an algorithm in the context of WSNs (time computation and bandwidth constraints) for synchronisation. Their solution has not been specifically applied for discussion scheduling.

In [10], the authors propose a technique called "Linear distance-based scheduling" of the sleep/running mode of every sensor to reduce power use. This technique is designed to work in a clustered network: the further from the cluster-head a sensor is, the greater the probability that this sensor is in sleep mode. Thus, the power use of every sensor depends on the distance to the cluster-head. This creates a discrepancy on the battery preservation between sensors, and leads to connectivity loss. As a conclusion, the authors propose to use a dynamic cluster formation to balance power consumption.

In [19], the authors address the problem of wake-up schedule in WSNs: 1-hop and 2-hop neighbors may interfere with sensor's communications. To avoid this, a sensor should avoid to use the same slot as those used by its 2-hop neigborhood. The authors propose to build a tree-based schedule in a distributed way for data collection.

In [11], the authors present a survey on optimisation techniques in several problems of WSN: Coverage problems, topology control, mobility, scheduling and routing. In particular, authors specify that in several investigations, time slot allocation problem (scheduling) is formulated as a graph coloring problem: two edges adjacent to the same sensor can not use the same time slot.

We propose in this paper a scheduling of the discussion between clusters. We express the different constraints between clusters as a mixed integer linear system. An introduction to mathematical programming can be found in [18]. In [15], the authors propose a general framework to deal with a wide class of periodic scheduling problems.

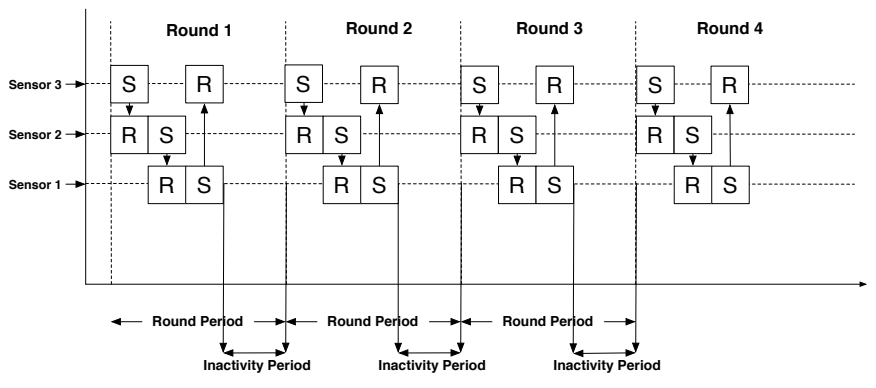

Fig. 2. Discussion protocol among sensors

\section{Models and Preliminary work}

\section{A. Network model}

We aim at managing communications over a WSN made up of $n$ sensors identified as $s_{1}, \ldots, s_{n}$.

We make the following assumptions:

- Each sensor has a unique identifier.

- The communication graph of the sensors is connected and undirected.

- Sending a message is instantaneous.

- All sensors are running at the same speed.

The system consists in $n$ independent sensors, communicating over a wireless medium. Such a Wireless Sensor Network can be represented as an undirected graph $G=(V, E)$, where $V$ is a set of sensors and $E$ a set of edges that represent possible communications between sensors: $(i, j)$ belongs to $E$ means that $i$ can communicate with $j$ (and conversely).

In a given graph $G=(V, E)$, we denote by $n=|V|$ the number of sensors in the network. Each sensor executes the same algorithm. The neighborhood $N_{i}$ of a sensor $i$ is defined as $N_{i}=\{j \in V \mid(i, j) \in E\}$. The number of neighbors of a sensor $i$ is called its degree, denoted by $\operatorname{deg}(i)$.

\section{B. Preliminary Works}

In this section, we present the 1-hop discussion protocol described in [5], [6].

\section{B.1 1-hop discussion protocol}

We proposed in [5] a light 1-hop discussion protocol over a clique of sensors. In this protocol, the communication relies on a ring communication structure. Each sensor has two slots for communication: one for receiving data (Slot R), and another one for sending data (Slot S). Sensors wake up periodically two by two so that, at a given time, there is at most one sensor sending data and another one receiving data. Fig. 2 describes the basic procedure of the protocol: during a period each sensor wakes up in such way as to receive information from its predecessor, and then deliver information to its successor. The remaining time is spent in sleep mode.

The protocol is set-up in a distributed way: sensors agree to establish the discussion in an ordered way. First, sensors synchronise, then they compute a schedule in the ring, that sums up to an ordering. This protocol is designed for a certain type of applications in which no alert needs to be reported urgently. This 


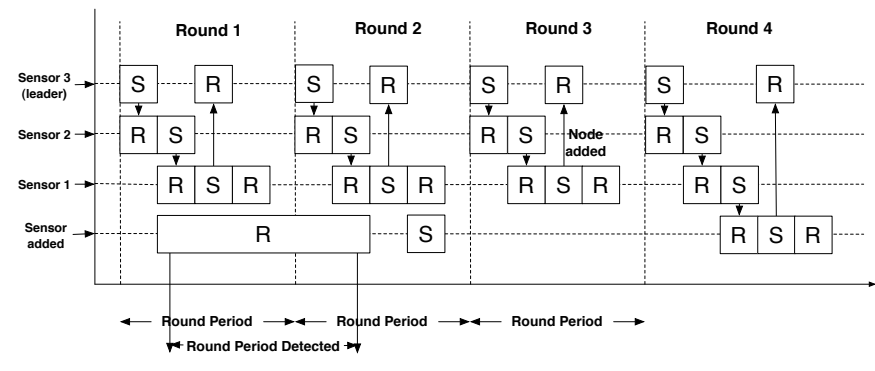

Fig. 3. Adding a sensor in the discussion

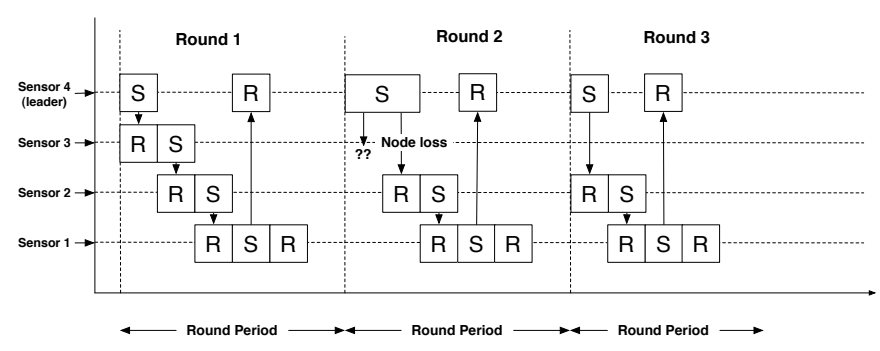

Fig. 4. Sensor crash

protocol avoids periodical negociation phases like in 802.15.4 protocol with beacon (used in ZigBee protocol).

\section{B.2 Refinement with the join/leaving procedure of sensors}

In [6], we have improved this protocol to allow the insertion or the deletion of sensors in the round discussion.

To integrate new sensors in the discussion, we use the remaining time period at the end of the round (the time period during which every sensor is sleeping). Fig. 3 summarizes the join process for a sensor.

During a first round, a joining sensor has to keep listening in order to detect several parameters of the discussion between sensors: round period length, last receiving slot,... Then, it announces at the end of the second round that it will join the network. As shown in Fig. 3, the last sensor slot is longer than others'. This additional amount of time is dedicated to listening sensors willing to join the network. Thus, in the second round, the last sensor is informed about a sensor connection, and waits for the third round to inform the first sensor about this connection. During the third round, all sensors are informed of the connection and, at the end of this round, the joining sensor is considered as a network member. Its slot will be larger than others in order to be able to manage the connection of an extra sensor. This method balances power use. Indeed, the last sensor of the ring consumes more energy than others, and the last sensor is the one that has joined the network the most recently (thus, it should have more energy than other sensors).

The updated protocol also handles sensor's disconnections. Fig. 4 summarizes the whole procedure when a sensor disconnects from the network.

When a sensor crashes, the discussion has to be updated to ensure a continuous information propagation. The disconnection will be detected with a basic acknowledgement mechanism: when an acknowledgement is missing, the sending sensor keeps awake until another sensor is able to receive the frame (obviously, the next uncrashed sensor in the ring). The slots assigned to all other sensors in the remaining discussion are then shifted. The parameters of the shift procedure depend on the number of crashed sensors and their location in the round discussion.

\section{Clustering for multi-hop WSN}

In this section, we propose a clustering algorithm allowing this token ring process to be implemented on each cluster. The schedule of the communication between clusters will be discussed in Section VI. An earlier version of this clustering technique can be found in [1].

\section{A. Specification of the clustering}

The token ring protocol presented in [5] requires a complete communication graph (i.e. a 1-hop WSN). Thus the clustering we propose computes cliques.

A sensor can be involved in several clusters: for instance, in Fig 5, 3 clusters have been computed and Sensors 2 and 4 belongs respectively to clusters $(C 1, C 2)$ and $(C 2, C 3)$. These sensors are called gateway sensors.

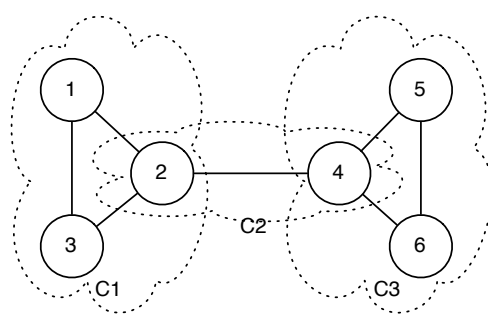

Fig. 5. A clique-clustered sensors network

Such sensors are involved in several rounds of 1-hop communications, more precisely one for each cluster they belong to. To schedule the discussion rounds between clusters, two adjacent clusters (i.e. clusters that share a set of common sensors) cannot be involved in communications at the same time, since it would imply message collisions. To take these constraints into account, the computed clustering is such that two adjacent sensors always belong to a common cluster, more precisely the edge between these two sensors has to be included in at least one cluster.

To limit collisions between clusters, and consequently the constraints in the scheduling, we build maximal cliques: a maximal clique is a clique that is included in no larger clique. The algorithm below computes all maximal cliques in the 2-hop neighborhood of a node, and then discards the cliques that are not necessary to cover all edges.

In order to deliver all the environmental data scanned by the sensors to the base station, the connectivity of the graph have to be maintained through the clustering procedure.

This gives the following requirements:

- Covering: every edge has to be covered by at least one cluster (so that constraints on communications of the two sensors are taken into account), 
- Connectivity: the graph composed by all sub-graphs of all clusters is connected,

- Maximal clique: all sensors in the same cluster are adjacent, and no other sensor is adjacent to all sensors in the cluster.

With these requirements and a correct schedule of the wakeup/sleeping state between clusters, the 1-hop discussion protocol can be extended to multi-hop.

\section{B. Clique-Clustering algorithm}

Each sensor computes cliques by exchanging messages, and locally decides the set of clusters to which it belongs. The result of the clustering procedure is a set of cluster $\mathcal{C}$.

The algorithm has three phases:

- The Setup Phase is the phase during which a sensor successively broadcasts its id and its neighborhood. When receiving this data from its neighbors, it updates local variables. At the end of this phase, each sensor knows its 2-hop neighborhood.

- The Clique Computation Phase is the phase when each sensor computes from its local variables all cliques to which it belongs.

- The Cleaning Phase takes place at the end and discards clusters $c$ such that all edges contained in $c$ also belong to another cluster of $\mathcal{C}$.

The three phases of the clustering procedure are detailed on the example given in Fig. 6.
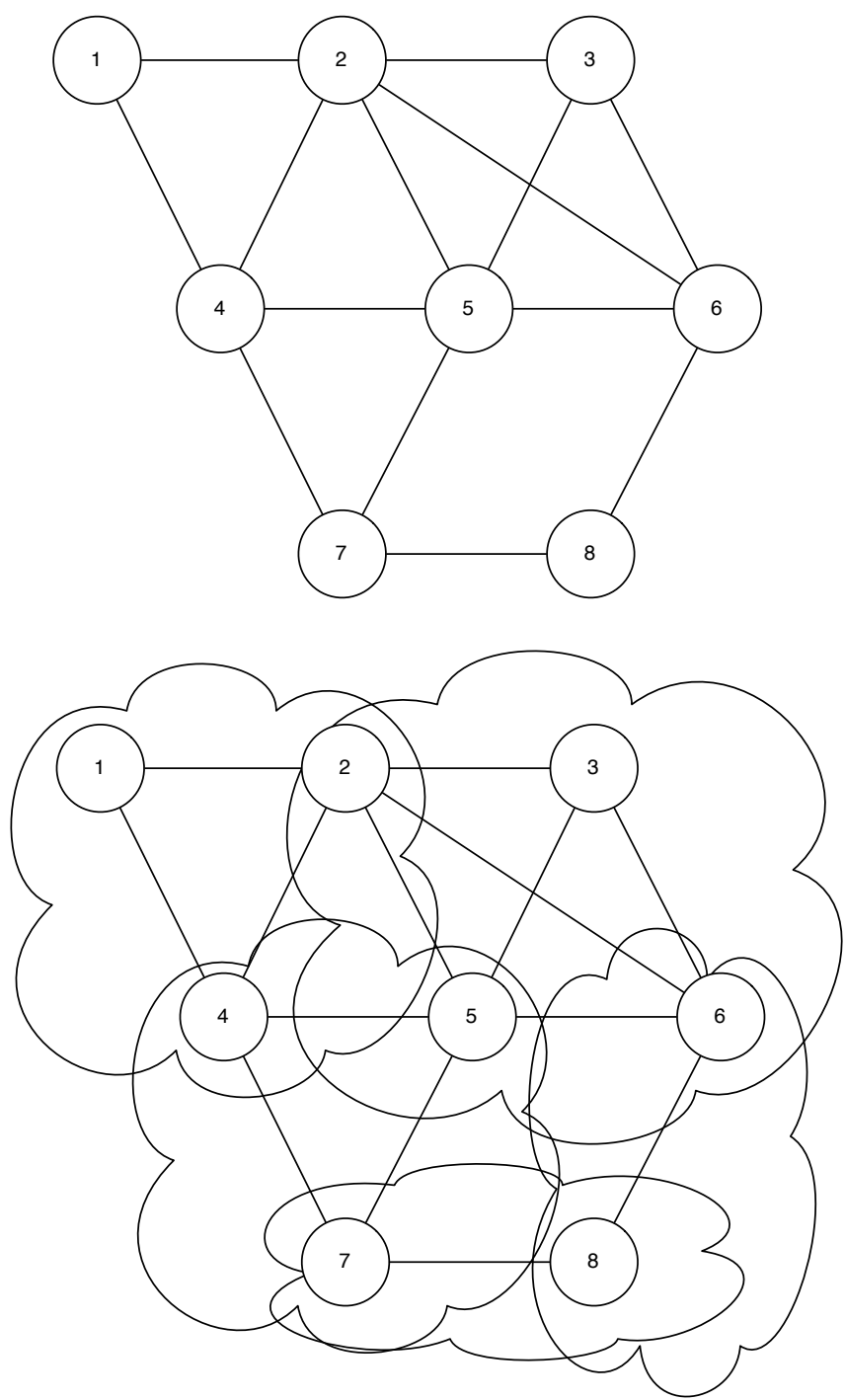

Fig. 6. A Wireless Sensor Network and its clustering 


\section{B.1 2-hop Neighborhood Computation}

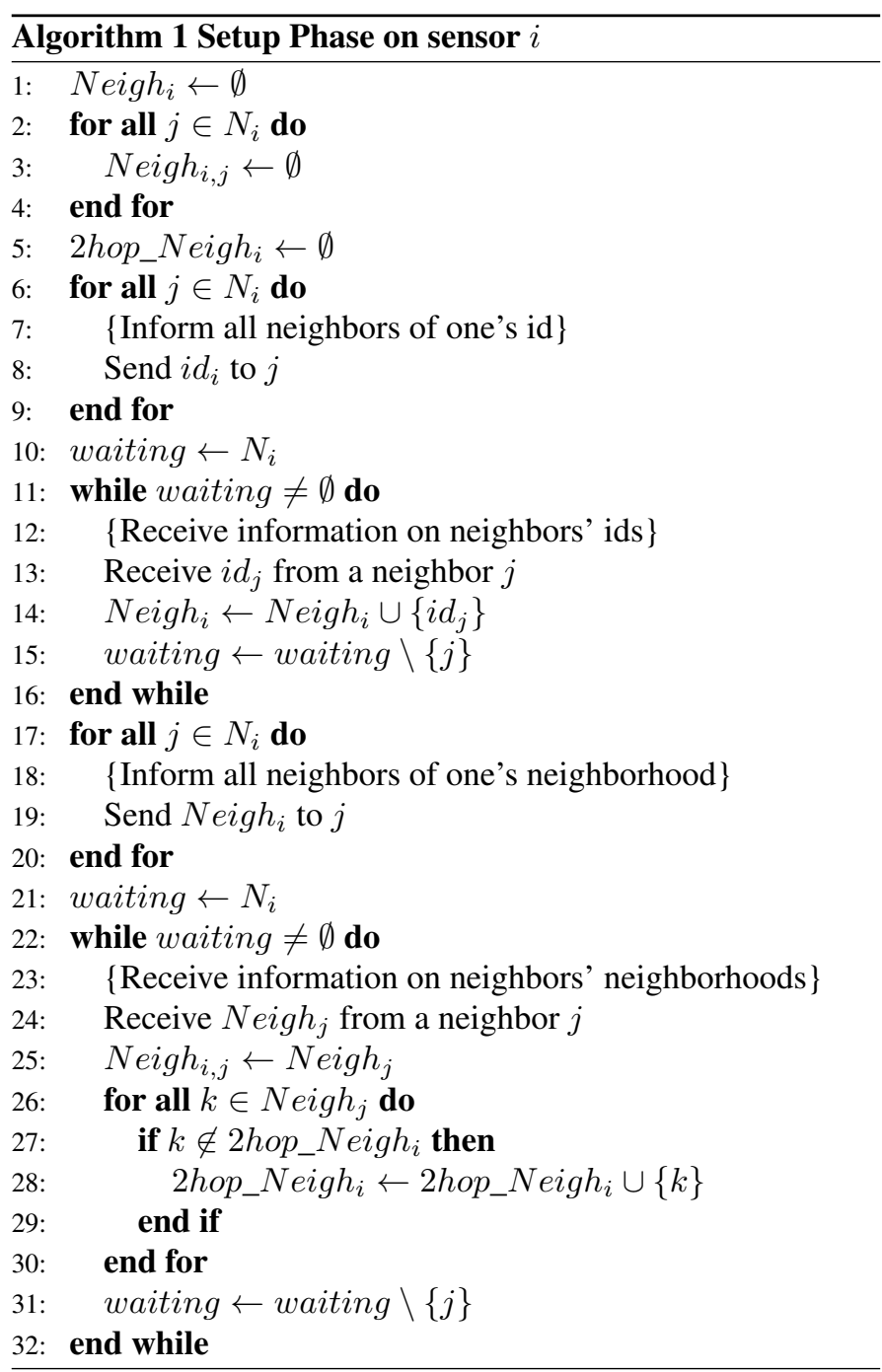

At the end of phase 1 (Algo. 1), we have the following results on the network given Fig. 6. We deliberately omit variables

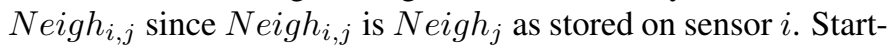
ing from then, all sensors know the ids of their neighbors, and we do not distinguish between a sensor and its id any longer.

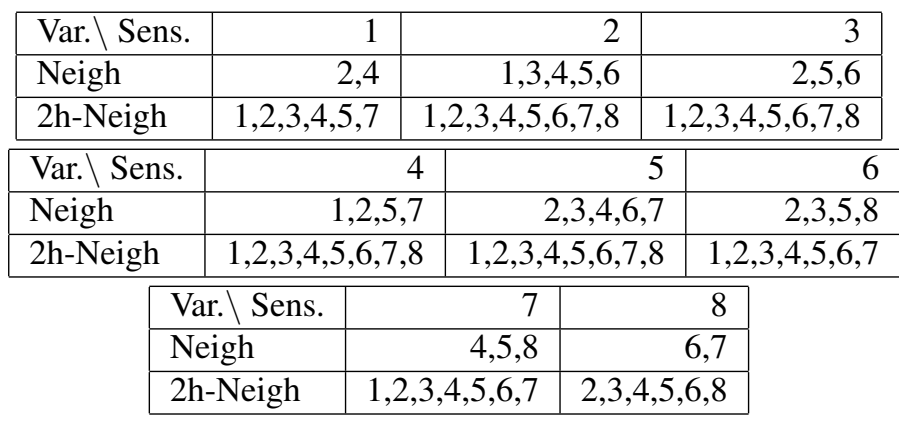

To compute its 2-hop neighborhood, sensors sends two messages (one with its id, and another with its 1-hop neighborhood) to its neighbors, and receives $2 \operatorname{deg}(i)$ messages from its neighbors.

\section{B.2 Cluster Computation}

Algorithm 2 computes maximal cliques by taking an edge $(j, k)$ in the 2-hop neighborhood and adding sensors $l$ in the 2-hop neighborhood of $i$ if they are adjacent to all sensors of the computed clique.

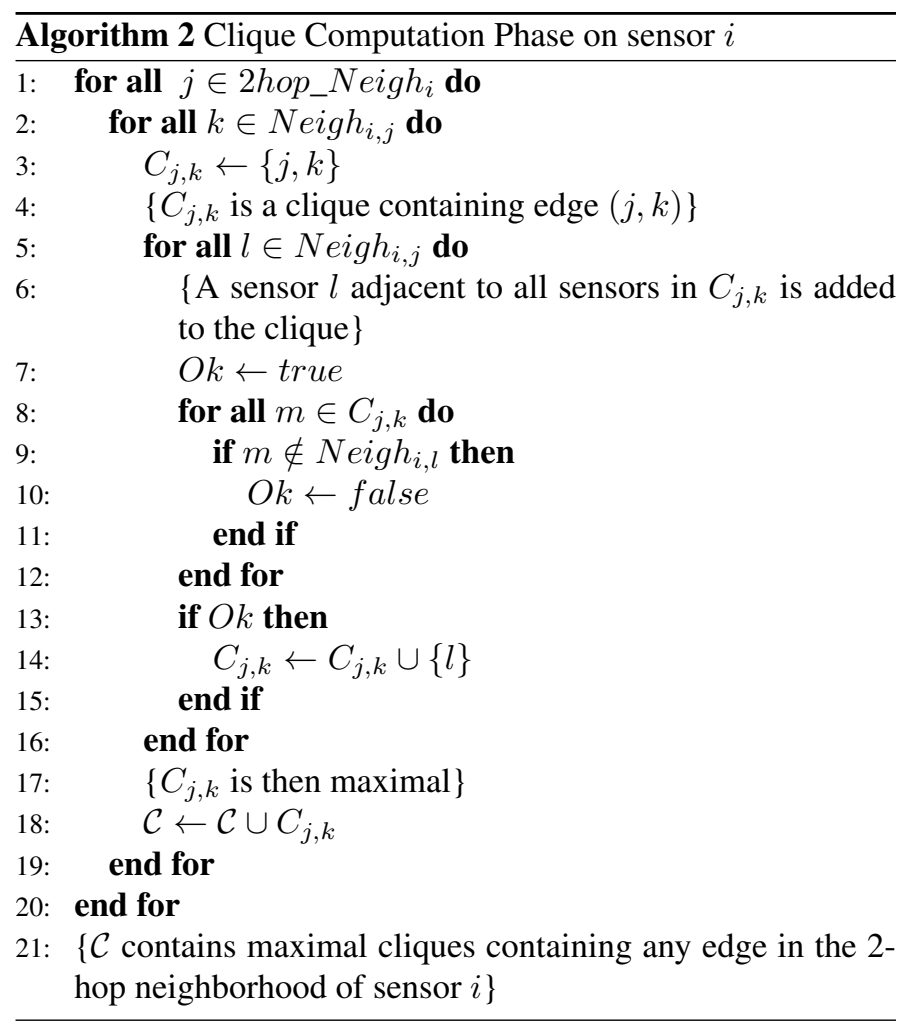

This algorithm computes all clusters to which the sensor belongs or to which it is adjacent (required for the cleaning phase). Since clusters must cover edges, the sensor computes a maximal clique $C_{j k}$ for each edge $(j, k)$ in its 2-hop neighborhood: it adds to the cluster each sensor $l$ adjacent to all sensors in the cluster.

At the end of the second phase of the clustering procedure on the example given Fig. $6, \mathcal{C}=\{\{1,2,4\},\{2,4,5\},\{2,3,5,6\}$, $\{4,5,7\},\{6,8\},\{7,8\}\}$.

Consider $\delta$ an upper bound on the degree of sensors. Since a clique cannot contain more than $\delta$ sensors (all sensors in a clique being neighbors), the outer loop has a range in $O\left(\delta^{2}\right)$ and each of the three other loops a range in $O(\delta)$. Hence, the complexity of this algorithm is $O\left(\delta^{5}\right)$.

\section{B.3 Cleaning Phase}

The cleaning phase aims at discarding clusters that cover no edge that is not covered by other clusters. Indeed, such clusters do not provide information on potential collisions that is not already known. Since clusters are maximal cliques, no cluster can be included in another one; but a cluster can be included in the union of several other clusters: such a cluster is discarded.

For instance, in the previous example, clique $\{2,4,5\}$ can be discarded since edges $(2,4),(2,5)$, and $(4,5)$ belong respectively to cliques $\{1,2,4\},\{2,3,5,6\}$ and $\{4,5,7\}$ 


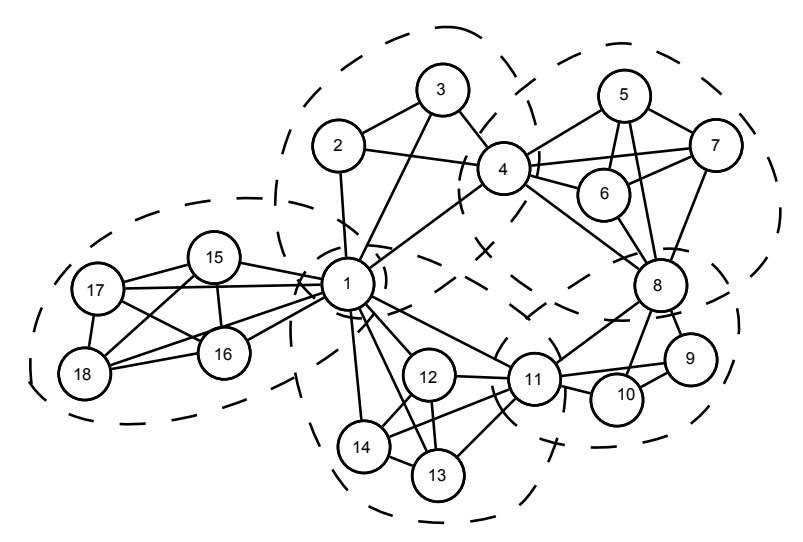

Fig. 7. Communication graph of a WSN

For every clique $C$ containing $i$, the algorithm checks if every pair of sensors $(l, m)$ (also an edge) is also part of another clique $D$. If this is the case, the clique is discarded.

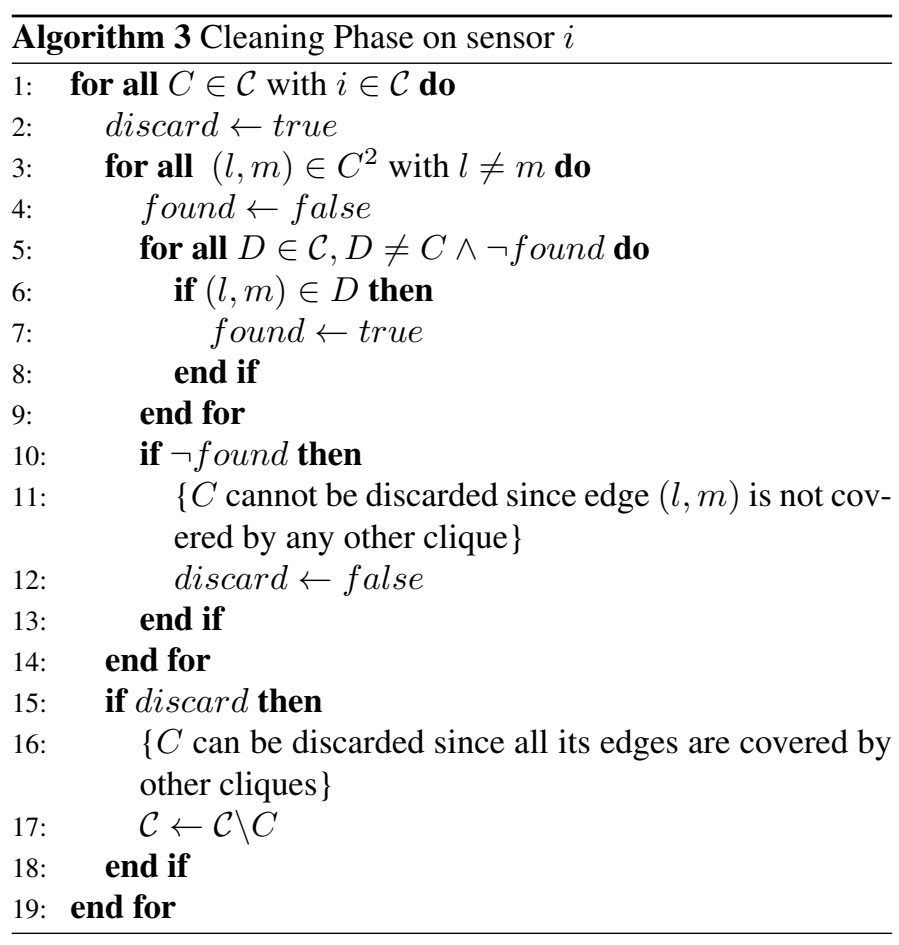

At the end of the clustering procedure, on the example given Fig. $6, \mathcal{C}=\{\{1,2,4\},\{2,3,5,6\},\{4,5,7\},\{6,8\},\{7,8\}\}$.

The complexity of this algorithm is upper-bounded by $O\left(\delta^{5}\right)$ $\left(O(\delta)\right.$ for loop at line 1, and $O\left(\delta^{2}\right)$ for loops at lines 3 and 5).

\section{Scheduling: an example}

The example below illustrates the result of the clustering, and the constraints it imposes on the scheduling of communications.

We consider the network presented in Fig. 7.

First, we apply the clustering algorithm presented in Section IV on this network. Computed clusters are $A=\{1,2,3,4\}$, $B=\{4,5,6,7,8\}, C=\{8,9,10,11\}, D=\{1,11,12,13,14\}$ and $E=\{1,15,16,17,18\}$.

We can notice that, in a cluster, the role of non-gateway sen-

\begin{tabular}{|c|c|c|c|c|c|c|c|c|c|c|c|}
\hline$n \backslash t$ & 1 & 2 & 3 & 4 & 5 & 6 & 7 & 8 & 9 & 10 & 11 \\
\hline 1 & $\mathrm{R}$ & $\mathrm{S}$ & & & $\mathrm{R}$ & $\mathrm{S}$ & & & & $\mathrm{R}$ & $\mathrm{S}$ \\
\hline 2 & & $\mathrm{R}$ & $\mathrm{S}$ & & & & & & & & \\
\hline 3 & $\mathrm{~S}$ & & & $\mathrm{R}$ & & & & & & & \\
\hline 4 & & & $\mathrm{R}$ & $\mathrm{S}$ & & & & $\mathrm{R}$ & $\mathrm{S}$ & & \\
\hline 5 & & & & & $\mathrm{~S}$ & & & & $\mathrm{R}$ & & \\
\hline 6 & & & & & & & $\mathrm{R}$ & $\mathrm{S}$ & & & \\
\hline 7 & & & & & & $\mathrm{R}$ & $\mathrm{S}$ & & & & \\
\hline 8 & $\mathrm{R}$ & $\mathrm{S}$ & & & $\mathrm{R}$ & $\mathrm{S}$ & & & & & \\
\hline 9 & & $\mathrm{R}$ & $\mathrm{S}$ & & & & & & & & \\
\hline 10 & $\mathrm{~S}$ & & & $\mathrm{R}$ & & & & & & & \\
\hline 11 & & & $\mathrm{R}$ & $\mathrm{S}$ & & & & $\mathrm{R}$ & $\mathrm{S}$ & & \\
\hline 12 & & & & & & $\mathrm{R}$ & $\mathrm{S}$ & & & & \\
\hline 13 & & & & & & & $\mathrm{R}$ & $\mathrm{S}$ & & & \\
\hline 14 & & & & & $\mathrm{~S}$ & & & & $\mathrm{R}$ & & \\
\hline 15 & & & & & & & & & $\mathrm{R}$ & $\mathrm{S}$ & \\
\hline 16 & & & & & & & & $\mathrm{R}$ & $\mathrm{S}$ & & \\
\hline 17 & & & & & & & $\mathrm{R}$ & $\mathrm{S}$ & & & \\
\hline 18 & & & & & & & $\mathrm{~S}$ & & & & $\mathrm{R}$ \\
\hline
\end{tabular}

Fig. 8. Scheduling on the network

\begin{tabular}{|c|c|c|c|c|}
\hline$n \backslash t$ & 1 & 2 & 3 & 4 \\
\hline 1 & $\mathrm{R}$ & $\mathrm{S}$ & & \\
\hline 2 & & $\mathrm{R}$ & $\mathrm{S}$ & \\
\hline 3 & $\mathrm{~S}$ & & & $\mathrm{R}$ \\
\hline 4 & & & $\mathrm{R}$ & $\mathrm{S}$ \\
\hline
\end{tabular}

Fig. 9. Scheduling of cluster A

\begin{tabular}{|c|c|c|c|c|}
\hline$n \backslash t$ & 1 & 2 & 3 & 4 \\
\hline 8 & $\mathrm{R}$ & $\mathrm{S}$ & & \\
\hline 9 & & $\mathrm{R}$ & $\mathrm{S}$ & \\
\hline 10 & $\mathrm{~S}$ & & & $\mathrm{~S}$ \\
\hline 11 & & & $\mathrm{R}$ & $\mathrm{S}$ \\
\hline
\end{tabular}

Fig. 10. Scheduling of cluster $\mathrm{C}$

sors is symmetric, i.e. the place of two non-gateway sensors of a cluster, like sensor 2 and sensor 3, can be exchanged.

A valid communication planning on this network is shown in Fig. 8.

In this example, cluster $A$ and cluster $C$ can communicate at the same time since they have no common sensor. Cluster $B$ and $D$ are in the same situation.

Here, we choose to place communications of cluster $A$ and $C$ first, then communications of clusters $B$ and $D$, and finally, communications of cluster $E$.

For cluster $A$, the first two time slots are reserved for communications of sensor 1 , which is a gateway sensor with cluster $D$. Similarly, we start communications of cluster $C$ with communications of gateway sensor 8 . Since sensors 4 and 8 are in cluster $B$, they cannot be involved in communications at the same time. Thus, sensor 4 can speak in cluster $A$ only at time 3 and 4 . For the same reason, sensor 11 can only communicate at time 3 and 4. This leads to the scheduling presented in figure 9 and 10.

We build the scheduling for cluster $B$ and $D$, presented in 11 


\begin{tabular}{|c|c|c|c|c|c|}
\hline$n \backslash t$ & 5 & 6 & 7 & 8 & 9 \\
\hline 4 & & & & $\mathrm{R}$ & $\mathrm{S}$ \\
\hline 5 & $\mathrm{~S}$ & & & & $\mathrm{R}$ \\
\hline 6 & & & $\mathrm{R}$ & $\mathrm{S}$ & \\
\hline 7 & & $\mathrm{R}$ & $\mathrm{S}$ & & \\
\hline 8 & $\mathrm{R}$ & $\mathrm{S}$ & & & \\
\hline
\end{tabular}

Fig. 11. Scheduling of cluster $B$

\begin{tabular}{|c|c|c|c|c|c|}
\hline$n \backslash t$ & 5 & 6 & 7 & 8 & 9 \\
\hline 1 & $\mathrm{R}$ & $\mathrm{S}$ & & & \\
\hline 11 & & & & $\mathrm{R}$ & $\mathrm{S}$ \\
\hline 12 & & $\mathrm{R}$ & $\mathrm{S}$ & & \\
\hline 13 & & & $\mathrm{R}$ & $\mathrm{S}$ & \\
\hline 14 & $\mathrm{~S}$ & & & & $\mathrm{R}$ \\
\hline
\end{tabular}

Fig. 12. Scheduling of cluster $\mathrm{D}$

\begin{tabular}{|c|c|c|c|c|c|}
\hline$n \backslash t$ & 7 & 8 & 9 & 10 & 11 \\
\hline 1 & & & & $\mathrm{R}$ & $\mathrm{S}$ \\
\hline 15 & & & $\mathrm{R}$ & $\mathrm{S}$ & \\
\hline 16 & & $\mathrm{R}$ & $\mathrm{S}$ & & \\
\hline 17 & $\mathrm{R}$ & $\mathrm{S}$ & & & \\
\hline 18 & $\mathrm{~S}$ & & & & $\mathrm{R}$ \\
\hline
\end{tabular}

Fig. 13. Scheduling of cluster $E$

and 12 in the same fashion. Since sensor 4 is involved in a communication at time 4 , sensors of cluster $B$ cannot communicate before time 5 , so do sensors of cluster $D$ because of sensor 11 .

Finally, we need to place communications of cluster $E$. Since sensors of cluster $D$ communicate until time slot 9 , sensor 1 cannot communicate until time slot 10 . So we build a planning for cluster $E$ with communication of sensor 1 in time slot 10 and 11 (figure 13).

The combination of all those plannings gives the solution shown in Fig. 8.

\section{Clusters communication scheduling}

Distant clusters can communicate at the same time. However, the existence of gateway sensors is an issue in planning communications, and forbids to apply the token algorithm straightforwardly. Indeed, if two clusters share a gateway, its communication according to the 1-hop protocol for any of these clusters prevents communications among sensors of the others: if a sensor has to listen to another given sensor, no other sensor within hearing distance must speak.

Making all sensors communicate one after the other is a valid communication scheme, but this scheduling is long and not scalable.

In this section, we model constraints on communications defining the validity of a schedule. First, we present decision variables, then we present constraints that define the 1-hop protocol on a cluster. After that, we present constraints that come from the existence of gateway sensors, and discuss the objective function of such a planning.

\section{A. Variables}

The scheduling is based on:

- $G=(V, E)$ : the communication graph of the system.

- $\mathcal{C}$ : set of clusters computed by the clustering algorithm (a cluster being a set of sensors).

We consider $T$ an upper bound on the optimal duration of a planning. Since we can build a valid communication scheduling with every sensor in every cluster speaking the one after the other, we choose $T=\sum_{c \in \mathcal{C}}|c|$ (with $|c|$ the number of sensors in $c$; considering that the time unit is one slot, $|c|$ is the time to achieve one round discussion in the cluster $c$ ).

We also define the following decision variables, the instantiation of which defines a scheduling:

- $\forall c \in \mathcal{C}, \forall t \in\{0, \ldots, T\}, \forall i \in C$,

$$
e_{i}^{c}(t)=\left\{\begin{array}{l}
1 \text { if } i \text { is in sending mode at time } t \text { for cluster } c \\
0 \text { else }
\end{array}\right.
$$

- $\forall c \in \mathcal{C}, \forall t \in\{0, \ldots, T\}, \forall i \in C$,

$$
r_{i}^{c}(t)=\left\{\begin{array}{l}
1 \text { if } i \text { in in reception mode at time } t \text { for cluster } c \\
0 \text { else }
\end{array}\right.
$$

- $\forall c \in \mathcal{C}, \forall i \in C$,

$$
s_{i}^{c}=\left\{\begin{array}{l}
1 \text { if } i \text { starts communication for cluster } c \\
0 \text { else }
\end{array}\right.
$$

We use the convention that $\forall c \in \mathcal{C}, \forall i \in c, \forall t \leqslant 0, e_{i}^{c}(t)=0$.

\section{B. Intracluster constraints}

Since the first sensor speaking in a cluster in a round is the cluster initiator, and since, according to the protocol described in section III-B, a sensor speaks just after listening (except for the initiator, that speaks first), variables $r_{i}^{c}(t)$ can be deduced from $e_{i}^{c}(t)$ and $s_{i}^{c}(t)$. When a non-initiator sensor $i$ is in reception mode, it is in sending mode in the following time slot. In this case, $s_{i}^{c}=0$ and $r_{i}^{c}(t)=e_{i}^{c}(t+1)$. If $i$ is initiator, it is in sending mode $|c|$ time slot before being in reception mode. In this case, $s_{i}^{c}=1$, and $e_{i}^{c}(t-|c|)=r_{i}^{c}(t)$.

Thus, $r_{i}^{c}(t)=\left(1-s_{i}^{c}\right) \times e_{i}^{c}(t+1)+s_{i}^{c} \times e_{i}^{c}(t-|c|)$.

Now, this equation is not linear, and to be able to use a MILP solver, we are willing to replace it with an equivalent set of linear equations or inequations.

First, since all quantities are nonnegative, we have:

$$
\left\{\begin{array}{l}
r_{i}^{c}(t) \geqslant\left(1-s_{i}^{c}\right) \times e_{i}^{c}(t+1) \\
r_{i}^{c}(t) \geqslant s_{i}^{c} \times e_{i}^{c}(t-|c|)
\end{array}\right.
$$

Since either $s_{i}^{c}(t)$ or $1-s_{i}^{c}(t)$ is zero, these inequations imply that $r_{i}^{c}(t) \geq\left(1-s_{i}^{c}\right) \times e_{i}^{c}(t+1)+s_{i}^{c} \times e_{i}^{c}(t-|c|)$, and since all sensors speak and listen exactly once in each of their cluster (constraints stated later will ensure this), the constraints above are equivalent to $r_{i}^{c}(t)=\left(1-s_{i}^{c}\right) \times e_{i}^{c}(t+1)+s_{i}^{c} \times e_{i}^{c}(t-|c|)$.

Now, for $a$ and $b$ in $\{0,1\}$, we have $a . b \geq a+b-1$. Thus

$$
\left\{\begin{array}{l}
r_{i}^{c}(t) \geqslant\left(1-s_{i}^{c}\right)+e_{i}^{c}(t+1)-1=e_{i}^{c}(t+1)-s_{i}^{c} \\
r_{i}^{c}(t) \geqslant s_{i}^{c}+e_{i}^{c}(t-|c|)-1
\end{array}\right.
$$

Conversely, the last equations are equivalent to the previous ones since $r_{i}^{c}(t) \geq 0$. 
This leads to the two following set of linear constraints:

$$
\forall c \in \mathcal{C}, \forall i \in c, \forall t \in\{0, \ldots, T\}, r_{i}^{c}(t) \geqslant e_{i}^{c}(t+1)-s_{i}^{c}
$$

$$
\forall c \in \mathcal{C}, \forall i \in c, \forall t \in\{0, \ldots, T\}, r_{i}^{c}(t) \geqslant s_{i}^{c}+e_{i}^{c}(t-|c|)
$$

There is at most one sensor in sending mode at a time in a cluster:

$$
\forall c \in \mathcal{C}, \forall t \in\{0, \ldots, T\}, \sum_{i \in c} e_{i}^{c}(t) \leqslant 1
$$

And there is at most one sensor in reception mode at a time in a cluster:

$$
\forall c \in \mathcal{C}, \forall t \in\{0, \ldots, T\}, \sum_{i \in c} r_{i}^{c}(t) \leqslant 1
$$

For a cluster $c$, all sensors in $c$ are in sender mode exactly once (for $c$ ) during a round, which means that for any sensor $i$, there is an unique time slot $t$ such that $i$ is in sending mode:

$$
\forall c \in \mathcal{C}, \forall i \in c, \sum_{t=0}^{T} e_{i}^{c}(t)=1
$$

And all sensors are also in receiver mode exactly once:

$$
\forall c \in \mathcal{C}, \forall i \in c, \sum_{t=0}^{T} r_{i}^{c}(t)=1
$$

When a sensor is in sending mode for a cluster, there is at least one sensor in reception mode for the cluster (if sensor $i$ is not sending data, i.e. $e_{i}^{c}(t)=0$, the constraint below is not restrictive, and constraint 4 already imposes that at most one sensor is listening in the cluster):

$$
\forall c \in \mathcal{C}, \forall i \in c, \forall t \in\{0, \ldots, T\}, e_{i}^{c}(t) \leqslant \sum_{j \in c} r_{j}^{c}(t)
$$

And similarly, when a sensor is in reception mode, there is at least one sensor in sending mode for the cluster:

$$
\forall c \in \mathcal{C}, \forall i \in c, \forall t \in\{0, \ldots, T\}, r_{i}^{c}(t) \leqslant \sum_{j \in c} e_{j}^{c}(t)
$$

\section{Intercluster constraints}

Intercluster constraints model the fact that, when a gateway sensor is speaking in one of its clusters, no adjacent sensor (i.e. no sensor in another of its clusters) can be listening; and that, on the other hand, when it is listening in a cluster, no adjacent sensor can be speaking.

When a sensor $i$ belongs to several clusters, if $i$ is in sending mode for one cluster, no sensor in the other clusters to which $i$ belongs can be in reception mode:

$$
\begin{array}{r}
\forall c \in \mathcal{C}, \forall i \in c, \forall t \in\{0, \ldots, T\}, \\
\sum_{\substack{c^{\prime} \neq c \\
c^{\prime} \ni i}} \sum_{j \in c^{\prime}} r_{j}^{c^{\prime}}(t) \leqslant\left(1-e_{i}^{c}(t)\right) \times n \times|\mathcal{C}|
\end{array}
$$

$n \times|\mathcal{C}|$ is obviously always greater than $\sum_{c^{\prime} \neq c, c^{\prime} \ni i} \sum_{j \in c^{\prime}}$ $r_{j}^{c^{\prime}}(t)$, since $r_{j}^{c^{\prime}}(t)$ is always less than 1. Thus, this constraint only imposes that when sensor $i$ is speaking in $c$ (i.e. $e_{i}^{c}(t)=1$ ), $\sum_{c^{\prime} \neq c, c^{\prime} \ni i} \sum_{j \in c^{\prime}} r_{j}^{c^{\prime}}(t)=0$, i.e. that all $r_{j}^{c^{\prime}}(t)=0$ (since $r_{j}^{c^{\prime}}(t) \geq 0$ ): thus no sensor $j$ in a cluster $c^{\prime}$ other than $c$ and to which $i$ belongs can be listening.

Similarly, if $i$ is in reception mode for one cluster, no sensor in other clusters to which $i$ belongs can be in sending mode:

$$
\begin{aligned}
& \forall c \in \mathcal{C}, \forall i \in c, \forall t \in\{0, \ldots, T\}, \\
& \sum_{c^{\prime} \neq c} \sum_{j \in c^{\prime}} e_{j}^{c^{\prime}}(t) \leqslant\left(1-r_{i}^{c}(t)\right) \times n \times|\mathcal{C}| \\
& c^{\prime} \ni i
\end{aligned}
$$

Constraints 1 through 10 define a communication scheduling such that all sensors listen and speak exactly once in each of their clusters, and no two communications can collide.

\section{Objective function and interpretation}

Any scheduling verifying the constraints 1 through 10 is valid. However, we are interested in computing an optimal scheduling according to some criterion. This criterion is expressed through an objective function, which we try to minimize (or to maximize).

We minimize the round length. This objective may for instance describe a network in which local information updates need to be as frequent as possible.

To describe this situation, we introduce a new variable $Y$, that is an upper bound on the duration of the scheduling. For any time $t$, if there is a $e_{i}^{c}(t) \neq 0, Y \geqslant t$. So, if $e_{i}^{c}(t)=1$, we have $Y \geqslant t \times e_{i}^{c}(t)$. Else, if $e_{i}^{c}(t)=0$, this constraint is $Y \geq 0$ which is always true.

This leads to the following constraints:

$\forall t \in\{0, \ldots, T\}, \forall c \in \mathcal{C}, \forall i \in c$,

$$
Y \geqslant t \times e_{i}^{c}(t)
$$

Then we search to minimize $Y$, the smallest upper bound, which is the length of the shortest planning. This leads to the objective function:

$$
\min \{Y\}
$$

Variables $e_{i}^{c}(t), r_{i}^{c}(t), s_{i}^{c}$ and $Y$, constraints 1 through 11 and the objective function $\min (Y)$ make up a mathematical program, that can be solved by integer linear programming. Solutions of such a program are computed by a solver like CPLEX for instance and give the shortest possible scheduling in a given network.

Solving a MILP is a NP-hard problem. Hence, this method requires (at worst) a number of elementary operations that is exponential in the number of variables and constraints. This centralized method (that is not designed to be implemented on sensors) computes an optimal planning and is designed to provide lower bounds on schedules that sensors may be able to compute. In particular, we intend to work on cheaper decentralized methods to compute sub-optimal schedules. 


\section{Conclusion}

In this paper, we investigate the computation of a planning of communications that allows a maximum of parallel communication on the top of a clustered multi-hop WSN. We develop a Mixed Integer Linear Program representing a centralized method to find such a scheduling. The specific clustering procedure that allows this scheduling is also detailed.

Such an optimal scheduling, though computed in a centralized fashion, is useful to provide benchmarks to which distributed solutions can be compared.

As future works, we intend to work on distributed methods to compute schedules that avoid message collisions: the whole network will organise itself by local arrangement. Through local organization, sensors have to design an efficient gathering of information until reaching the base station. In particular, an arborescent hierarchization of the schedule will be investigated.

\section{REFERENCES}

[1] K. Abdurusul,T. Bernard and H. Fouchal, An Efficient Multi-Hop MAC Protocol for WSN, Proceedings of the 17th International Symposium on Computers and Communications, IEEE CS Press, 2012.

[2] Y. M. Akkaya Kemal, A survey on routing protocols for wireless sensor networks, Ad Hoc Networks, vol. 3, no. 5, pp. 325-349, 2005

[3] J. Ben-Othman and B. Yahya, Energy efficient and qos based routing protocol for wireless sensor networks, Journal of Parallel and Distributed Computing, vol. 70, no. 8, pp. 849-857, 2010.

[4] J. Ben-Othman, K. Bessaoud, A. Bui and L. Pilard, Self-stabilizing algorithm for efficient topology control in Wireless Sensor Networks, Journal of Computationnal Sciences, vol. 4, no. 4, pp. 199-208, 2013.

[5] T. Bernard and H. Fouchal, Slot Assignment Over Wireless Sensor Networks, Proceedings of the 24th IEEE International Conference on Global Communications, pp 1-5, IEEE CS Press, 2011.

[6] T. Bernard and H. Fouchal, A low Energy Consumption Mac Protocol for WSN, Proceedings of the IEEE International Conference on Communications, pp 533-537, IEEE CS Press, 2012.

[7] M. Buettner, G. Yee, E. Anderson,R. Han, X-mac: A short preamble mac protocol for duty-cycled wireless sensor networks, Proceedings of the 4th International Conference on Embedded Networked Sensor Systems,pp 307320, ACM Press, 2006.

[8] A. da Cunha and D. da Silva Jr. An approach for the reduction of power consumption in sensor nodes of wireless sensor networks: Case analysis of mica2, Proceedings of 6th Workshop on Embedded Computer Systems: Architectures, MOdeling, and Simulation, LNCS 4017, pp 132-141, SpringerVerlag, 2006.

[9] J.H. Chang and L. Tassiulas, Maximum Lifetime Routing in Wireless Sensor Networks, IEEE/ACM Transactions on Networking, vol.12, no. 4, pp 609-619, 2004

[10] J. Deng, Y. Han, W. Heinzelman and P. Varshney, Scheduling Sleeping Nodes in High Density Cluster-based sensor networks, Journal Mobile Networks and Applications, vol. 10, no. 6, pp.825-835, 2005

[11] A. Gogu, D. Nace, A. Dilo and N. Mertnia, Optimization Problems in Wireless Sensor Networks, Proceedings of the International Conference on Complex, Intelligent and Software Intensive Systems, pp. 302-309, IEEE CS, 2011

[12] A. Gogu, D. Nace and Y. Challal, A Framework for Solving the Configuration Problem in Wireless Sensor Networks Proceedings of the 8th International Conference on Broadband and Wireless Computing, Communication and Applications, pp. 64-60, IEEE, 2013

[13] A. Djouama, L. Mokdad, and M. Abdennebi, Performance evaluation of lifetime-driven admission control for infrastructure-less clustered wireless networks, Concurrency and Computation : Practice and Experience, vol. 25 , no. 5, pp. 718-727, 2013

[14] I. Rhee, A. Warrier, M. Aia, and J. Min, Z- mac: An hybrid mac for wireless sensor networks, Proceedings of the 3rd International Conference on Embedded Networked Sensor Systems, pp 90-101, ACM Press, 2005.

[15] P. Serafini, W. Ukovich, A Mathematical Model for Periodic Scheduling Problems, SIAM J. Discret. Math., vol. 2, no. 4 pp 550-581, Society for Industrial and Applied Mathematics, 1989.

[16] M.L. Sichitiu and C. Veerarittiphan, Simple, accurate time synchronization for wireless sensor networks, Proceedings of the Wireless Communications and Networking Conference, pp 1266-1273 vol.2, IEEE CS Press, 2003
[17] Y. Wei, J. Heidemann, and D. Estrin, An energy-efficient mac protocol for wireless sensor networks, Proceedings of the 21th Annual Joint Conference of the IEEE Computer and Communications Societies INFOCOM, pp 15671576, IEEE CS Press, 2002.

[18] L. Wolsey, Integer Programming, Wiley, 1998

[19] F.J. Wu and Y.C. Tseng, Distributed Wake-up Scheduling for Data Collection in Tree-based Wireless Sensor Networks, IEEE Communications Letters, vol. 13, no. 11, pp. 850-852, IEEE, 2009

[20] B. Yahya and J. Ben-Othman, Towards a Classification of Energy-Aware MAC Protocols for Wireless Sensor Networks, Wireless Communications and Mobile Computing, vol. 12, no. 3, pp. 1572-1607, 2009. 